\title{
Adaptive Control of a Quadrotor UAV Transporting a Cable-Suspended Load with Unknown Mass
}

\author{
Shicong Dai, Taeyoung Lee, and Dennis S. Bernstein
}

\begin{abstract}
We design an adaptive controller for a quadrotor UAV transporting a point-mass payload connected by a flexible cable modeled as serially-connected rigid links. The mass of the payload is uncertain. The objective is to transport the payload to a desired position while aligning the links along the vertical direction from an arbitrary initial condition. A fixedgain nonlinear proportional-derivative controller is presented to achieve the desired performance for a nominal payload mass, and a retrospective cost adaptive controller is used to compensate for the payload mass uncertainty.
\end{abstract}

\section{INTRODUCTION}

Quadrotor unmanned aerial vehicles have the desirable capabilities of hovering and vertical take-off and landing. They have been envisaged for various applications, including mobile sensor network, aerial photography, and educational research. In particular, several aggressive maneuvers have been demonstrated by utilizing their high thrust-to-weight ratio [1]-[3].

Autonomous aerial transportation of a cable-suspended load has been studied traditionally for helicopters [4], [5]. Small-size single or multiple autonomous vehicles are considered for load transportation and deployment [6]-[8]. However, these results are based on a simplified dynamic model, where the dynamic effects of the payload on the quadrotor are approximated by unstructured disturbances without considering the dynamic coupling between the payload and the quadrotor. As such, they may not be suitable for rapid load transportation, where the dynamics of the payload can be excited significantly.

A coordinate-free form of the equations of motion is developed in [9] for the integrated dynamics of a quadrotor, cable, and payload. Based on this complete dynamic model, geometric tracking control systems are constructed for a single quadrotor transporting a cable-suspended point mass [9], and for a cooperative group of quadrotors transporting a common payload connected by multiple cables, while controlling the relative formation among them [10]. It is further generalized to the case where a payload is connected to a quadrotor via a flexible cable [11].

Shicong Dai is with School of Automation Science and Electrical Engineering, Beihang University, Beijing, 100191 daibluewater@gmail.com

Taeyoung Lee is with Department of Mechanical and Aerospace Engineering, The George Washington University, Washington, DC 20052 tyleedgwu. edu

Dennis S. Bernstein is with Department of Aerospace Engineering, University of Michigan, Ann Arbor, MI 48109 dsbaero@umich .edu

* This research has been supported in part by NSF under the grant CMMI1243000 (transferred from 1029551), CMMI-1335008, and CNS-1337722.

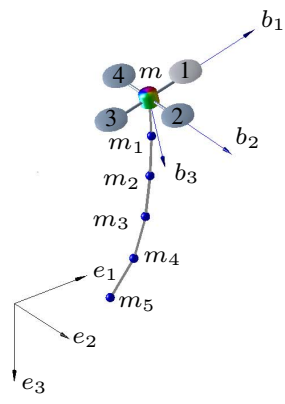

Fig. 1. Quadrotor UAV with a cable-suspended load. The cable is modeled as a serial connection of an arbitrary number of links (We show 5 links here as an example). It is assumed that the mass of the payload is unknown.

These approaches incorporate the dynamic coupling between the payload and the quadrotor explicitly in the control system design and stability analysis. However, it is assumed that the mass of the payload is exactly known. If various payloads with a broad range of mass properties must be delivered at low cost, then it is desirable that the quadrotor transports the payload autonomously without relying on the exact knowledge of the payload mass.

The objective of this paper is to design an adaptive control system for a quadrotor that delivers a cable-suspended payload without knowledge of the payload mass. The cable is modeled as serially-connected rigid links and the payload is modelled as a point-mass. A fixed-gain geometric nonlinear PD controller is first presented to achieve desired performance for a nominal payload mass. Retrospective cost adaptive control (RCAC) is then constructed to compensate for the payload mass uncertainty.

RCAC is a direct adaptive control technique that requires limited modeling information [12]-[14]. The algorithm has been applied to various dynamic systems, such as aircraft [14], multiple linkages [15], [16], and spacecraft attitude dynamics [17]. RCAC is based on optimizing the retrospective performance, which re-optimizes the current controller based on past data. This strategy enables RCAC to control a system based on a simplified input-output relation instead of a detailed state-space model. In this paper, RCAC uses components of the impulse response of the quadrotor with a fixed-gain controller in the loop.

\section{Dynamic Model of A QuAdrotor With A FLEXIBLE CABLE}

Consider a quadrotor UAV with a payload that is connected by a chain of $n$ links, as illustrated at Fig. 1. The inertial frame is defined by the unit vectors $e_{1} \triangleq\left[\begin{array}{lll}1 & 0 & 0\end{array}\right]^{\mathrm{T}}$, $e_{2} \triangleq\left[\begin{array}{lll}0 & 1 & 0\end{array}\right]^{\mathrm{T}}$, and $e_{3} \triangleq\left[\begin{array}{lll}0 & 0 & 1\end{array}\right]^{\mathrm{T}} \in \mathbb{R}^{3}$, and the third axis $e_{3}$ 
corresponds to the direction of gravity. Define a body-fixed frame $\left\{\vec{b}_{1}, \vec{b}_{2}, \vec{b}_{3}\right\}$ whose origin is located at the center of mass of the quadrotor, and its third axis $\vec{b}_{3}$ is aligned with the axis of symmetry of the quadrotor.

The location of the mass center, and the attitude of the quadrotor are denoted by $x \triangleq\left[\begin{array}{lll}x_{1} & x_{2} & x_{3}\end{array}\right]^{\mathrm{T}} \in \mathbb{R}^{3}$ and $R \in \mathrm{SO}$ (3), respectively, where the special orthogonal group is $\mathrm{SO}(3) \triangleq\left\{R \in \mathbb{R}^{3 \times 3} \mid R^{T} R=I_{3}, \quad \operatorname{det} R=1\right\}$. A rotation matrix represents the linear transformation of a representation of a vector from the body-fixed frame to the inertial frame.

The dynamic model of the quadrotor is identical to [1]. The mass and the inertia matrix of the quadrotor are denoted by $m \in \mathbb{R}$ and $J \in \mathbb{R}^{3 \times 3}$, respectively. The quadrotor can generate a thrust $-f R e_{3} \in \mathbb{R}^{3}$ relative to the inertial frame, where $f \in \mathbb{R}$ is the total thrust magnitude. It also generates a moment $M \triangleq\left[\begin{array}{lll}M_{1} & M_{2} & M_{3}\end{array}\right]^{\mathrm{T}} \in \mathbb{R}^{3}$ relative to the bodyfixed frame. The pair $(f, M)$ is considered as the control input of the quadrotor.

Let $q_{i} \in \mathrm{S}^{2}$ be the unit vector representing the direction of the $i$-th link, measured outward from the quadrotor toward the payload, where the two-sphere is the manifold of unitvectors in $\mathbb{R}^{3}$, i.e., $\mathrm{S}^{2} \triangleq\left\{q \in \mathbb{R}^{3} \mid\|q\|=1\right\}$. For simplicity, we assume that the mass of each link is concentrated at the outboard end of the link, and the point where the first link is attached to the quadrotor corresponds to the mass center of the quadrotor. The mass and length of the $i$-th link are defined by $m_{i}$ and $l_{i} \in \mathbb{R}$, respectively. Thus, the mass of the payload corresponds to $m_{n}$, which is unknown. The corresponding configuration manifold of this system is $\mathbb{R}^{3} \times \mathrm{SO}(3) \times\left(\mathrm{S}^{2}\right)^{n}$.

To derive the kinematics equations, let $\Omega \triangleq$ $\left[\begin{array}{lll}\Omega_{1} & \Omega_{2} & \Omega_{3}\end{array}\right]^{\mathrm{T}} \in \mathbb{R}^{3}$ be the angular velocity of the quadrotor represented relative to the body-fixed frame, and let $\omega_{i} \in \mathbb{R}^{3}$ be the angular velocity of the $i$-th link represented relative to the inertial frame. The angular velocity is normal to the direction of the link, i.e., $q_{i} \cdot \omega_{i}=0$. The kinematics equations are given by

$$
\begin{aligned}
\dot{R} & =R \hat{\Omega}, \\
\dot{q}_{i} & =\omega_{i} \times q_{i}=\hat{\omega}_{i} q_{i},
\end{aligned}
$$

where the hat map $\hat{:}: \mathbb{R}^{3} \rightarrow \mathfrak{s o}(3)$ is defined such that $\hat{x} y=x \times y$ for $x, y \in \mathbb{R}^{3}$, and the lie algebra is defined as $\mathfrak{s o}(3) \triangleq\left\{A \in \mathbb{R}^{3 \times 3} \mid A=-A^{T}\right\}$. The inverse of the hat map is denoted by $\vee: \mathfrak{s o}(3) \rightarrow \mathbb{R}^{3}$. The 2-norm of a matrix $A$ is denoted by $\|A\|$, and the dot product is denoted by $x \cdot y=x^{T} y$.

\section{A. Euler-Lagrange equations [11]}

For a quadrotor with a cable-suspended payload, the EulerLagrange equations on $\mathbb{R}^{3} \times \mathrm{SO}(3) \times\left(\mathrm{S}^{2}\right)^{n}$ are given by

$$
\begin{gathered}
J \dot{\Omega}+\hat{\Omega} J \Omega=M, \\
m_{00} \ddot{x}+\sum_{i=1}^{n} m_{0 i} \ddot{q}_{i}=-f R e_{3}+m_{00} g e_{3},
\end{gathered}
$$

$$
\begin{aligned}
& m_{i i} \ddot{q}_{i}-\hat{q}_{i}^{2}\left(m_{i 0} \ddot{x}+\sum_{\substack{j=1 \\
j \neq i}}^{n} m_{i j} \ddot{q}_{j}\right) \\
= & -m_{i i}\left\|\dot{q}_{i}\right\|^{2} q_{i}-\sum_{a=i}^{n} m_{a} g l_{i} \hat{q}_{i}^{2} e_{3},
\end{aligned}
$$

where the inertia values are given by

$$
\begin{gathered}
m_{00}=m+\sum_{i=1}^{n} m_{i}, \quad m_{0 i}=\sum_{a=i}^{n} m_{a} l_{i}, \quad m_{i 0}=m_{0 i}, \\
m_{i j}=\left(\sum_{a=\max \{i, j\}}^{n} m_{a}\right) l_{i} l_{j} .
\end{gathered}
$$

\section{B. Actuator Model}

The actuator model is identical to [1]. The total thrust $f$ and the total moment $M \triangleq\left[\begin{array}{lll}M_{1} & M_{2} & M_{3}\end{array}\right]^{\mathrm{T}}$ can be written as

$$
\left[\begin{array}{c}
f \\
M_{1} \\
M_{2} \\
M_{3}
\end{array}\right]=\left[\begin{array}{cccc}
1 & 1 & 1 & 1 \\
0 & -d & 0 & d \\
d & 0 & -d & 0 \\
-c_{\tau f} & c_{\tau f} & -c_{\tau f} & c_{\tau f}
\end{array}\right]\left[\begin{array}{c}
f_{1} \\
f_{2} \\
f_{3} \\
f_{4}
\end{array}\right],
$$

where $d$ is the distance from the mass center of the quadrotor to the center of each rotor in the $\vec{b}_{1}, \vec{b}_{2}$ plane, $c_{\tau f}$ is a fixed constant, and $f_{i}$ is the thrust of the $\mathrm{i}$-th propeller.

\section{RCAC Algorithm StATEMENT}

Consider the MIMO system

$$
\begin{aligned}
x(k+1) & =A x(k)+B u(k)+D_{1} w(k), \\
z(k) & =C x(k)+D_{2} w(k),
\end{aligned}
$$

where $x(k) \in \mathbb{R}^{l_{x}}, z(k) \in \mathbb{R}^{l_{z}}, u(k) \in \mathbb{R}^{l_{u}}$, and $w(k) \in$ $\mathbb{R}^{l_{w}}$.

For $n_{\mathrm{f}}>0, z(k)$ is given by

$$
\begin{aligned}
z(k)= & C A^{n_{\mathrm{f}}} x\left(k-n_{\mathrm{f}}\right)+D_{2} w(k)+\sum_{i=1}^{n_{\mathrm{f}}} C A^{i-1} D_{1} w(k-i) \\
& +\sum_{i=1}^{n_{\mathrm{f}}} H_{i} u(k-i)
\end{aligned}
$$

where $H_{i} \triangleq C A^{i-1} B$.

The signal $w(k)$ represents either disturbances to be rejected or commands to be followed, or both. The goal is to develop an adaptive output feedback controller that minimizes the performance variable $z$ in the presence of the unknown exogenous signal $w(k)$ with limited modeling information about (8) and (9). The required modeling data is described below.

\section{A. Control Law}

We use a strictly proper time-series controller of order $n_{\mathrm{c}}$ of the form

$u(k)=\mathcal{M}_{0}(k)+\sum_{i=1}^{n_{\mathrm{c}}} \mathcal{M}_{i}(k) u(k-i)+\sum_{i=1}^{n_{\mathrm{c}}} \mathcal{N}_{i}(k) z(k-i)$, 
where $\mathcal{M}_{0}(k) \in \mathbb{R}^{l_{u}}$ and, for all $i=1, \ldots, n_{\mathrm{c}}, \mathcal{M}_{i}(k) \in$ $\mathbb{R}^{l_{u} \times l_{u}}$ and $\mathcal{N}_{i}(k) \in \mathbb{R}^{l_{u} \times l_{z}}$. The controller (11) can be represented as

$$
u(k)=\theta(k) \phi(k),
$$

where

$$
\begin{aligned}
& \theta(k) \triangleq\left[\begin{array}{lll}
\mathcal{N}_{1}(k) & \cdots & \mathcal{N}_{n_{\mathrm{c}}}(k)
\end{array}\right. \\
& \left.\mathcal{M}_{1}(k) \quad \cdots \quad \mathcal{M}_{n_{\mathrm{c}}}(k) \mathcal{M}_{0}\right] \in \mathbb{R}^{l_{u} \times\left(n_{\mathrm{c}}\left(l_{u}+l_{z}\right)+1\right)}, \\
& \phi(k) \triangleq\left[\begin{array}{lll}
z^{\mathrm{T}}(k-1) & \cdots & z^{\mathrm{T}}\left(k-n_{\mathrm{c}}\right)
\end{array}\right. \\
& \left.u^{\mathrm{T}}(k-1) \quad \cdots \quad u^{\mathrm{T}}\left(k-n_{\mathrm{c}}\right) \quad 1\right]^{\mathrm{T}} \in \mathbb{R}^{n_{\mathrm{c}}\left(l_{u}+l_{z}\right)+1} .
\end{aligned}
$$

\section{B. Retrospective Performance}

For $\hat{\theta} \in \mathbb{R}^{l_{u} \times n_{\mathrm{c}}\left(l_{u}+l_{z}\right)+1}$ and $n_{\mathrm{f}} \geq 1$, we define the retrospective performance

$$
\hat{z}(\hat{\theta}, k) \triangleq z(k)+\sum_{i=1}^{n_{\mathrm{f}}} H_{i}[\hat{u}(k)-u(k-i)],
$$

where

$$
\hat{u}(k) \triangleq \hat{\theta} \phi(k)
$$

is the retrospective control. The retrospective performance $\hat{z}(\hat{\theta}, k)$ can be interpreted as the performance assuming that $\hat{\theta}$ was used in the past.

Defining $\Theta(k)=\operatorname{vec} \theta(k) \in \mathbb{R}^{n_{c} l_{u}\left(l_{z}+l_{u}\right)+l_{u}}$ and $\hat{\Theta}=$ $\operatorname{vec} \hat{\theta} \in \mathbb{R}^{n_{\mathrm{c}} l_{u}\left(l_{z}+l_{u}\right)+l_{u}}$, it follows that

$$
\begin{aligned}
\hat{z}(\hat{\Theta}, k) & \triangleq z(k)+\sum_{i=1}^{n_{\mathrm{f}}} \Phi^{\mathrm{T}}(k)[\hat{\Theta}-\Theta(k-i)] \\
& =z(k)-\sum_{i=1}^{n_{\mathrm{f}}} \Phi^{\mathrm{T}}(k) \Theta(k-i)+\Psi^{\mathrm{T}}(k) \hat{\Theta},
\end{aligned}
$$

where, for all $i=1, \ldots, n_{\mathrm{f}}, \Phi_{i}(k) \triangleq \phi(k-i) \otimes$ $H_{i}^{\mathrm{T}} \in \mathbb{R}^{\left(n_{\mathrm{c}} l_{u}\left(l_{z}+l_{u}\right)+l_{u}\right) \times l_{z}}$ and $\Psi(k) \triangleq \sum_{i=1}^{n_{\mathrm{f}}} \Phi_{i}(k) \in$ $\mathbb{R}^{\left(n_{c} l_{u}\left(l_{z}+l_{u}\right)+l_{u}\right) \times l_{z}}$.

\section{Cumulative Retrospective Cost Optimization}

The cumulative retrospective cost function is defined by

$$
\begin{aligned}
J(\hat{\Theta}, k) \triangleq & \sum_{j=0}^{k} \lambda^{k-i} \hat{z}(\hat{\Theta}, k)^{\mathrm{T}} R_{z} \hat{z}(\hat{\Theta}, k) \\
& +\lambda^{k}(\hat{\Theta}-\Theta(0))^{\mathrm{T}} R_{\Theta}(\hat{\Theta}-\Theta(0)),
\end{aligned}
$$

where $R_{\Theta} \in \mathbb{R}^{\left(n_{c} l_{u}\left(l_{z}+l_{u}\right)+l_{u}\right) \times\left(n_{c} l_{u}\left(l_{z}+l_{u}\right)+l_{u}\right)}$ and $R_{z} \in$ $\mathbb{R}^{l_{z}}$ are positive definite, and $\lambda \in(0,1]$ is the forgetting factor.

Let $P(0)=R_{\Theta}^{-1}$ and $\Theta(0) \in \mathbb{R}^{\left(n_{\mathrm{c}} l_{u}\left(l_{z}+l_{u}\right)+l_{u}\right)}$. Then, for all $k \geq 0$, the unique global minimizer of (16) is given by $\hat{\Theta}=\bar{\Theta}(k)$, where

$$
\begin{aligned}
& \Theta(k+1)=\Theta(k)-\frac{P(k) \Psi(k) \hat{z}(\Theta(k), k)}{\lambda R_{z}^{-1}+\Psi^{\mathrm{T}}(k) P(k) \Psi(k)}, \\
& P(k+1)=\frac{1}{\lambda}\left[P(k)-\frac{P(k) \Psi(k) \Psi^{\mathrm{T}}(k) P(k)}{\lambda R_{z}^{-1}+\Psi^{\mathrm{T}}(k) P(k) \Psi(k)}\right] .
\end{aligned}
$$

\section{RCAC FOR QUADROTOR TRANSPORTING A CABLE-SUSPENDED LOAD}

Let $x_{\mathrm{p}, \mathrm{d}} \in \mathbb{R}^{3}$ be the fixed desired location of the payload. We assume that all of the states of (1)-(5) are measured. The control objective is to move a payload of uncertain mass $m_{n}$ to $x_{\mathrm{p}, \mathrm{d}}$ while aligning the links in the vertical direction. Note that the position of the payload is given by $x_{\mathrm{p}} \triangleq\left[x_{\mathrm{p}, 1} x_{\mathrm{p}, 2} x_{\mathrm{p}, 3}\right]=x+\sum_{i=1}^{n} l_{i} q_{i}$.

Since $x_{\mathrm{p}, 1}$ and $x_{\mathrm{p}, 2}$ are not linearly controllable for the total force $f$ and the moment $M$ in the hanging equilibrium, the controller of a quadrotor is separated into a trajectory control loop and an attitude control loop.

To achieve the control objective, the trajectory control loop directly uses the total thrust as the command to be designed. A simplified dynamic model is first derived for the design of the trajectory control loop. In this loop, a PD controller is designed to make the hanging equilibrium asymptotically stable for $x, \dot{x}, q_{i}$ and $\omega_{i}$ with a nominal payload mass. The RCAC controller is then designed to compensate for the payload mass uncertainty.

To orient the propellers in the direction of the thrust command given by the trajectory control loop, the attitude control loop uses $f$ and $M$ as the command to be designed. In this loop, a geometric nonlinear PD controller is designed to achieve asymptotic tracking of the thrust command. The control architecture is given by Figure 2 .

\section{A. Simplified Dynamic Model}

For the given equations of motion (4), the control thrust is given by $-f R e_{3}$. This implies that the total thrust magnitude $f$ can be arbitrarily chosen within a total thrust bound, but the direction of the thrust vector is always along the third body-fixed axis. Also, the rotational attitude dynamics of the quadrotor are not affected by the translational dynamics of the quadrotor or the dynamics of the links. Therefore, we replace the term $-f R e_{3}$ in (4) with $F \triangleq\left[\begin{array}{lll}F_{1} & F_{2} & F_{3}\end{array}\right]^{\mathrm{T}} \in \mathbb{R}^{3}$, as shown in (19). Note that the simplified dynamics (19) are independent of the quadrotor attitude dynamics given by (3). This is equivalent to separating the dynamics into an outer loop, where the attitude $R$ is assumed to be instantaneously achievable, and an inner loop that controls the attitude $R$. The simplified dynamics are given by (5) and

$$
m_{00} \ddot{x}+\sum_{i=1}^{n} m_{0 i} \ddot{q}_{i}=F+m_{00} g e_{3} .
$$

\section{B. PD Trajectory Controller}

In this subsection, a fixed-gain PD trajectory controller is designed to make states of the simplified dynamics converge to the hanging equilibrium, where $x=x_{\mathrm{p}, \mathrm{d}}-\sum_{i=1}^{n} l_{i} e_{3}$, $\dot{x}=0_{3 \times 1}$ and, for all $i \leq n, \omega_{i}=0_{3 \times 1}$ and $q_{i}=e_{3}$. Note that the PD trajectory controller is designed for a nominal payload mass. We define the nominal payload mass as $\bar{m}_{n}$, and define the payload mass uncertainty as $\tilde{m}_{n} \triangleq m_{n}-\bar{m}_{n}$. 


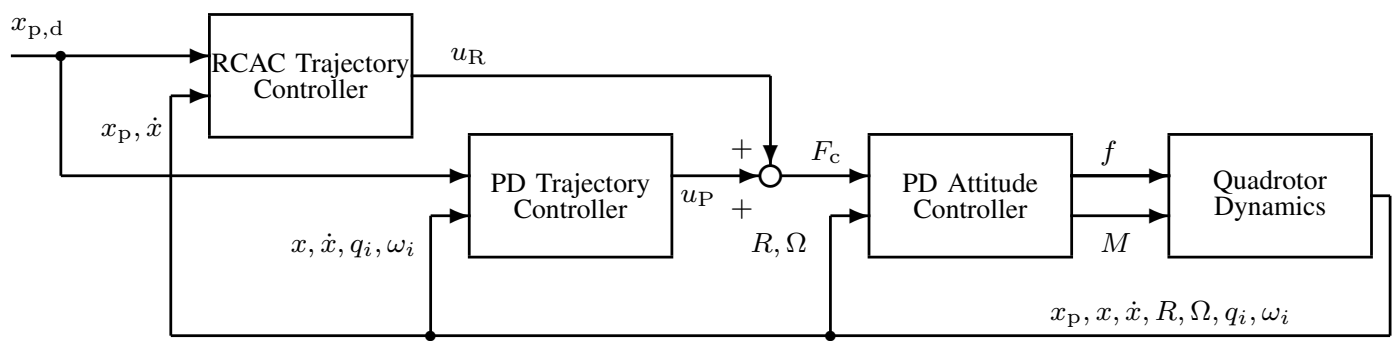

Fig. 2. Closed-loop quadrotor system. The measurements needed by the control system are $x_{\mathrm{p}}, x, \dot{x}, R, \Omega, q_{i}$, and $\omega_{i}$, for all $i \leq n$. Note that $x_{\mathrm{p}}$ can be calculated from $x$ and $q_{i}$ using knowledge of the length of each link. $x_{\mathrm{p}}$ and $\dot{x}$ are used for feedback in the RCAC trajectory controller. $x, \dot{x}, q_{i}$, and $\omega_{i}$ for all $i \leq n$ are used for feedback in the PD trajectory controller. $R$ and $\Omega$ are used for feedback in the PD attitude controller.

The control from the PD trajectory controller is given by

$$
\begin{aligned}
u_{\mathrm{P}}= & -K_{x} e_{x}-K_{\dot{x}} e_{\dot{x}}-\sum_{i=1}^{n} K_{\omega_{i}} e_{\omega_{i}} \\
& -\sum_{i=1}^{n} K_{q_{i}} e_{q_{i}}-\left(m_{00}-\tilde{m}_{n}\right) g e_{3},
\end{aligned}
$$

where

$$
\begin{gathered}
e_{x} \triangleq x-x_{\mathrm{p}, \mathrm{d}}+\sum_{i=1}^{n} l_{i} e_{3}, \quad e_{\dot{x}} \triangleq \dot{x}, \\
e_{\omega_{i}} \triangleq\left[\begin{array}{ll}
e_{1} & e_{2}
\end{array}\right]^{T} \omega_{i}, \quad e_{q_{i}} \triangleq\left[\begin{array}{ll}
e_{1} & e_{2}
\end{array}\right]^{T}\left(e_{3} \times q_{i}\right) .
\end{gathered}
$$

Note that $m_{00}-\tilde{m}_{n}$ is the total mass of the quadrotor and links with a nominal payload mass.

\section{RCAC Trajectory Controller}

In this subsection, an RCAC trajectory controller is designed to compensate for the payload mass uncertainty. Since RCAC is used to control thrust only in the $z$-axis direction, the control is given by $u_{\mathrm{R}}(k) \triangleq\left[\begin{array}{lll}0 & 0 & u_{\mathrm{R}, 3}(k)\end{array}\right]^{\mathrm{T}}$. We define the performance vector for RCAC as

$$
z(k) \triangleq\left[\begin{array}{c}
\tilde{x}_{\mathrm{p}, 3}(k) \\
\dot{x}_{3}(k)
\end{array}\right] \in R^{2},
$$

where $\tilde{x}_{\mathrm{p}, 3}$ is the third entry of the payload position error

$$
\tilde{x}_{\mathrm{p}}(k) \triangleq x_{\mathrm{p}}(k)-x_{\mathrm{p}, \mathrm{d}} .
$$

The parameters $H_{i}$ for RCAC are obtained from the impulse response of the simplified dynamics and the PD controller given by (5), (19), and (20) with $\tilde{m}_{n}=0$. The initial condition is given by $x(0)=x_{\mathrm{p}, \mathrm{d}}-\sum_{i=1}^{n} l_{i} e_{3}, \dot{x}(0)=0_{3 \times 1}$, for all $j, q_{j}(0)=e_{3}$ and $\omega_{j}(0)=0_{3 \times 1}$. Specifically, for all $k \leq n_{\mathrm{f}}$, we define

$$
H_{k} \triangleq z(k),
$$

where $z(k) \in \mathbb{R}^{2}$ is the column performance vector with the unit impulse input to the third channel with the PD trajectory controller given by (20) in the loop. Thus, the input used to obtain $z(k)$ can be given by $F_{\mathrm{c}}(0)=u_{\mathrm{P}}(0)+e_{3}, F_{\mathrm{c}}(1)=$ $u_{\mathrm{P}}(1), F_{\mathrm{c}}(2)=u_{\mathrm{P}}(2), \ldots, F_{\mathrm{c}}\left(n_{\mathrm{f}}\right)=u_{\mathrm{P}}\left(n_{\mathrm{f}}\right)$. This is shown in Figure 3.

The thrust command $u_{\mathrm{R}, 3}(k) \in \mathbb{R}$ is calculated from the RCAC algorithm given by (12), (15), (17), and (18).

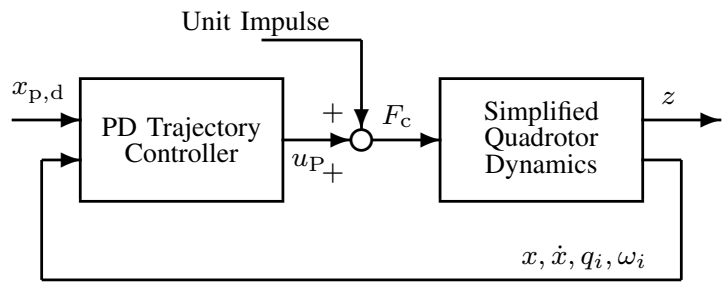

Fig. 3. Method for obtaining $H_{i}$. Note that the PD trajectory controller is in the loop. $x, \dot{x}, q_{i}$, and $\omega_{i}$ for all $i \leq n$ are used for feedback in the PD trajectory controller. Note that the attitude controller and quadrotor dynamics are replaced by the simplified dynamics given by (5) and (19).

\section{Attitude Controller}

In this subsection, a fixed-gain geometric nonlinear PD controller is designed to asymptotically follow the total thrust command $F_{\mathrm{c}} \in \mathbb{R}^{3}$. The thrust command on the step $k$ is given by

$$
F_{\mathrm{c}}=u_{\mathrm{R}}(k)+u_{\mathrm{P}}(k) .
$$

The desired direction of the third body-fixed axis is

$$
b_{3_{d}}=-\frac{F_{\mathrm{c}}}{\left\|F_{\mathrm{c}}\right\|} .
$$

There is an additional one-dimensional degree of freedom of the quadrotor attitude that corresponds to the rotation about the third body-fixed axis. To resolve it, the desired direction of the first body-fixed axis, namely, $b_{1_{d}} \in \mathrm{S}^{2}$ is chosen to be the initial first body-fixed axis $b_{1_{0}}$.

The corresponding desired attitude is chosen as

$$
R_{\mathrm{d}}=\left[\begin{array}{lll}
-\frac{\hat{b}_{3_{d}}^{2} b_{1_{d}}}{\left\|\hat{b}_{3_{d}}^{2} b_{1_{d}}\right\|} & \frac{\hat{b}_{3_{d}} b_{1_{d}}}{\left\|\hat{b}_{3_{d}} b_{1_{d}}\right\|} & b_{3_{d}}
\end{array}\right],
$$

which is in $\mathrm{SO}(3)$. The desired angular velocity is obtained by the attitude kinematics equation

$$
\Omega_{d}=\left(R_{\mathrm{d}}^{T} \dot{R}_{\mathrm{d}}\right)^{\vee} .
$$

Next, we define the tracking error variables for the attitude and the angular velocity as

$$
\begin{aligned}
& e_{R} \triangleq \frac{1}{2}\left(R_{\mathrm{d}}^{T} R-R^{T} R_{\mathrm{d}}\right)^{\vee}, \\
& e_{\Omega} \triangleq \Omega-R^{T} R_{\mathrm{d}} \Omega_{d} .
\end{aligned}
$$


The thrust magnitude command and moment command vector of the quadrotor are chosen as

$$
\begin{aligned}
f_{c}= & -F_{c} \cdot R e_{3}, \\
M_{c}= & -K_{R} e_{R}-K_{\Omega} e_{\Omega}+\Omega \times J \Omega \\
& -J\left(\hat{\Omega} R^{T} R_{\mathrm{d}} \Omega_{d}-R^{T} R_{\mathrm{d}} \dot{\Omega}_{d}\right),
\end{aligned}
$$

where $K_{R} \triangleq \omega_{n}^{2}\|J\|$ and $K_{\Omega} \triangleq 2 \omega_{n} \zeta\|J\|$, where $\omega_{n}$ and $\zeta$ are positive.

\section{NUMERICAL EXAMPLES}

The sample time for each example is chosen as $0.01 \mathrm{sec}$. Properties of the quadrotor are chosen as

$$
m=0.5 \mathrm{~kg}, \quad J=\operatorname{diag}\left[\begin{array}{lll}
0.557 & 0.557 & 1.05
\end{array}\right] \times 10^{-2} \mathrm{kgm}^{2} .
$$

Three identical links with $n=3, m_{i}=0.1 \mathrm{~kg}$, and $l_{i}=$ $0.2 \mathrm{~m}$ are considered in the case with a nominal payload mass. The payload mass uncertainty is $\tilde{m}_{3}=0.1 \mathrm{~kg}$, thus $m_{3}=0.2 \mathrm{~kg}$ with the actual payload mass. Four rotors with maximum thrust $5 \mathrm{~N}, d=0.2 \mathrm{~m}$, and $c_{\tau f}=0.01 \mathrm{~m}$ are used as actuators.

The desired location of the payload is selected as $x_{\mathrm{p}, \mathrm{d}}=$ $0_{3 \times 1}$. The initial conditions for the quadrotor are given by

$$
\begin{gathered}
x(0)=\left[\begin{array}{ccc}
0.6 & -0.7 & 0.2
\end{array}\right]^{\mathrm{T}}, \dot{x}(0)=0_{3 \times 1}, \\
R(0)=I_{3}, \quad \Omega(0)=0_{3 \times 1} .
\end{gathered}
$$

The initial directions of the links are chosen such that the cable is curved along the horizontal direction, as illustrated at Figure 4, and the initial angular velocity of each link is chosen as zero.

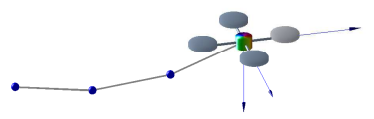

Fig. 4. Initial state of the links.

The chosen parameters of the RCAC trajectory controller are

$$
n_{c}=2, n_{\mathrm{f}}=30, R_{\Theta}=0.1 I_{7}, R_{z}=\operatorname{diag}[10.1], \lambda=1 .
$$

The chosen parameters of the PD trajectory controller are

$$
\begin{aligned}
& K_{x}=I_{3}, K_{\dot{x}}=\operatorname{diag}\left[\begin{array}{lll}
1.75 & 1.75 & 1.67
\end{array}\right], \\
& K_{q_{1}}=\left[\begin{array}{cc}
0 & -4.8 \\
4.8 & 0 \\
0 & 0
\end{array}\right], K_{\omega_{1}}=\left[\begin{array}{cc}
0 & -0.6 \\
0.6 & 0 \\
0 & 0
\end{array}\right] \text {, } \\
& K_{q_{2}}=\left[\begin{array}{cc}
0 & 3.8 \\
-3.8 & 0 \\
0 & 0
\end{array}\right], K_{\omega_{2}}=\left[\begin{array}{cc}
0 & -0.02 \\
0.02 & 0 \\
0 & 0
\end{array}\right] \text {, } \\
& K_{q_{3}}=\left[\begin{array}{cc}
0 & -0.2 \\
0.2 & 0 \\
0 & 0
\end{array}\right], K_{\omega_{3}}=\left[\begin{array}{cc}
0 & -0.006 \\
0.006 & 0 \\
0 & 0
\end{array}\right] \text {. }
\end{aligned}
$$

The parameters of the PD attitude controller are chosen as

$$
\omega_{n}=10 \mathrm{rad} / \mathrm{sec}, \zeta=0.707 \text {. }
$$

A saturation block is added for the moment command. The amplitude limit for $M_{1}$ and $M_{2}$ is chosen as $1 \mathrm{~N}-\mathrm{m}$, and the amplitude limit for $M_{3}$ is chosen as $0.01 \mathrm{~N}-\mathrm{m}$.

To show the stabilizing performance for the links, we define the error functions

$$
e_{q} \triangleq \sum_{i=1}^{n}\left\|q_{i}-e_{3}\right\|, \quad e_{\omega} \triangleq \sum_{i=1}^{n}\left\|\omega_{i}\right\| .
$$

\section{A. PD controller}

In this example, the RCAC controller is turned off. Therefore $F_{c}(k)=u_{\mathrm{P}}(k)$. Figure 5 shows that the closed-loop is stable and the links are aligned in the vertical direction, but there is an asymptotic error of the payload position about 1 $\mathrm{m}$ along the $z$-axis.
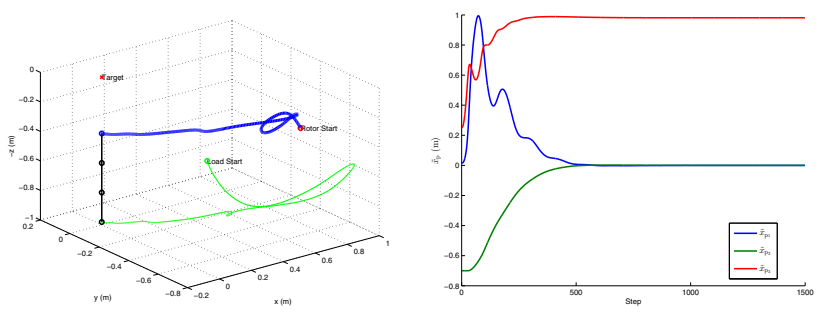

(a) 3D trajectory of the quadrotor (b) Position error $\tilde{x}_{\mathrm{p}}$ of the payload translation (thick) and the payload (thin)

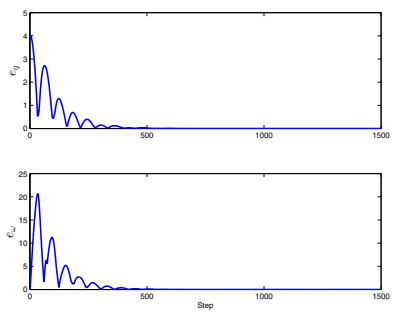

(c) Direction error $e_{q}$ and angular velocity error $e_{\omega}$ for links

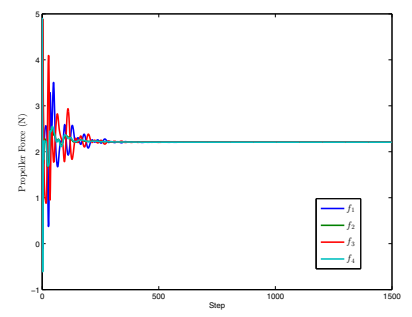

(d) Thrust for each propeller
Fig. 5. PD example. (a) shows that the closed-loop is stable. (b) shows that there is a constant asymptotic error of the payload position about $1 \mathrm{~m}$ along the $z$-axis for $\tilde{m}_{n}=0.1 \mathrm{~kg}$. The asymptotic error is due to the extra gravity provided by the positive payload mass uncertainty. Nevertheless, (c) shows that the links are asymptotically aligned in the vertical direction and stop swinging in about 4 sec.

\section{B. PID controller}

Since there is a large asymptotic error along the $z$-axis in the example with the PD controller alone, we add an extra integral term in the force command in this example to deal with the asymptotic error. Thus,

$$
F_{c}(k)=u_{\mathrm{P}}(k)-\sum_{i=0}^{k}\left[\begin{array}{ccc}
0 & 0 & 0 \\
0 & 0 & 0 \\
0 & 0 & 0.25
\end{array}\right] \tilde{x}_{\mathrm{p}}(k) T_{\mathrm{s}},
$$

where $\tilde{x}_{\mathrm{p}}(k)$ is given by (22) and $T_{\mathrm{s}}$ is the sample time. Figure 6 shows that the asymptotic error along the $z$-axis is slowly eliminated. Figure 6(a) shows that the payload first arrives below the desired position and then moves upward to the desired position. 

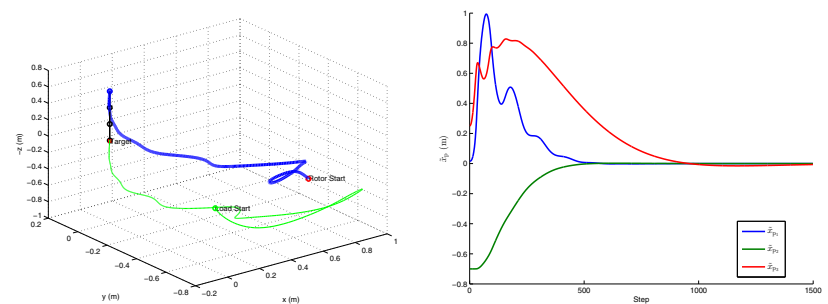

(a) $3 \mathrm{D}$ trajectory of the quadrotor (b) Position error $\tilde{x}_{\mathrm{p}}$ of the payload translation (thick) and the payload (thin)

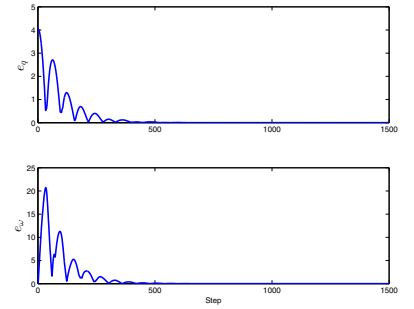

(c) Direction error $e_{q}$ and angular velocity error $e_{\omega}$ for links

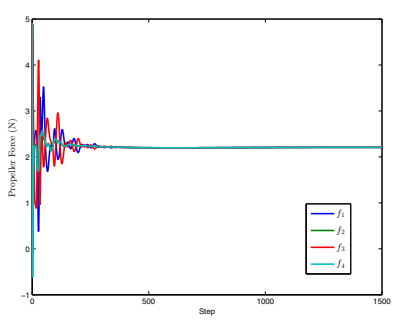

(d) Thrust for each propeller
Fig. 6. PID example. (a) shows that the payload first arrives below the desired position and then moves upward to the desired position (b) shows that compared with the case with the PD controller alone, the asymptotic error of the payload position along the $z$-axis is eliminated. (c) shows that the links are asymptotically aligned in the vertical direction and stop swinging in about $4 \mathrm{sec}$ as in the PD example.

\section{RCAC controller $+P D$ Controller}

In this example, the RCAC controller is turned on, and thus $F_{c}(k)=u_{\mathrm{P}}(k)+u_{\mathrm{R}}(k)$. Figure 7 shows that the asymptotic error along the $z$-axis is eliminated. Compared with the case with the PID controller, the error of the payload position along the $z$-axis converges faster and the payload does not approach the desired position from below but rather from the same horizontal plane.

\section{CONCLUSION}

We design a tracking controller for a quadrotor UAV with a point-mass payload connected by a flexible cable modeled as serially-connected rigid links. A fixed-gain geometric nonlinear PD controller is first presented to achieve desired performance for a nominal payload mass. Enabled by the impulse response with the PD controller in the loop, a retrospective cost adaptive controller is designed to compensate for the payload mass uncertainty in the case of aggressive maneuvers. Compared with the performance of the fixedgain controller with an integral control term, the adaptive controller has smaller settling time and overshoot.

\section{REFERENCES}

[1] T. Lee, M. Leok, and N. McClamroch, "Geometric tracking control of a quadrotor UAV on SE(3)," in Proc. CDC, 2010, pp. 5420-5425.

[2] J. Gillula, H. Huang, M. Vitus, and C. Tomlin, "Design of guaranteed safe maneuvers using reachable sets: Autonomous quadrotor aerobatics in theory and practice," in Proc. ICRA, 2010, pp. 1649-1654.

[3] D. Mellinger, N. Michael, and V. Kumar, "Trajectory generation and control for precise aggressive maneuvers with quadrotors," Int. J. Robotics Research, vol. 31, no. 5, pp. 664-674, 2012.

[4] L. Cicolani, G. Kanning, and R. Synnestvedt, "Simulation of the dynamics of helicopter slung load systems," J. Am. Helicopter Soc., vol. 40, no. 4, pp. 44-61, 1995.

[5] M. Bernard, "Generic slung load transportation system using small size helicopters," in Proc. ICRA, 2009, pp. 3258-3264.

[6] I. Palunko, P. Cruz, and R. Fierro, "Agile load transportation," IEEE Robot. Autom. Mag., vol. 19, no. 3, pp. 69-79, 2012.
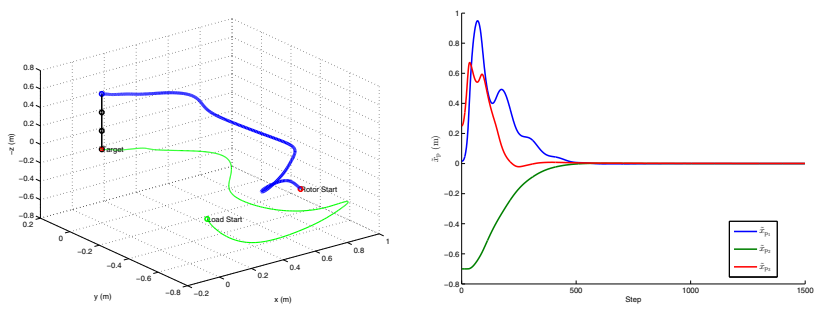

(a) 3D trajectory of the quadrotor (b) Position error $\tilde{x}_{\mathrm{p}}$ of the payload translation (thick) and the payload (thin)

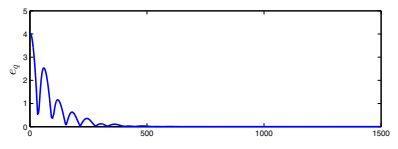

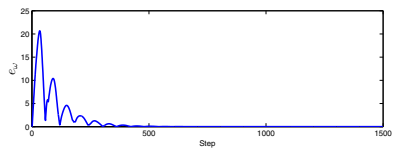
velocity error $e_{\omega}$ for links

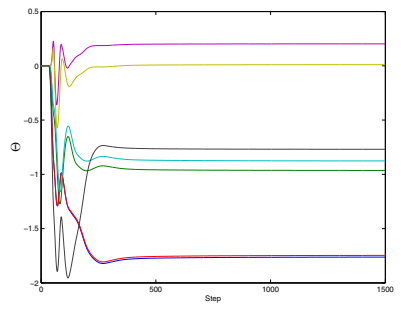

(e) Controller parameter $\Theta(k)$ (c) Direction error $e_{q}$ and angular

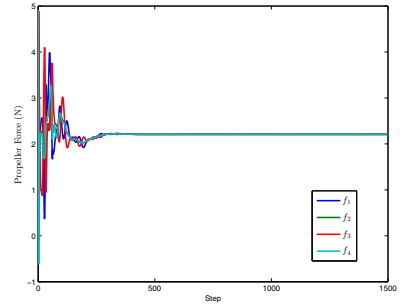

(d) Thrust for each propeller

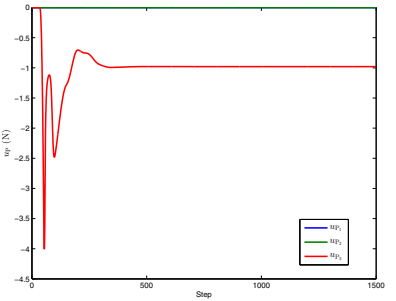

(f) Force command from the RCAC trajectory controller $u_{\mathrm{R}}$
Fig. 7. PD+RCAC example. (a) shows the payload does not approach the desired position from below but rather from the same horizontal plane. (b) shows that compared with the case with the PID controller, the error of the payload position along the $z$-axis in this case converges faster. (c) shows that the links are asymptotically aligned in the vertical direction and stop swinging in about $4 \mathrm{sec}$ as in the PD and PID examples.

[7] N. Michael, J. Fink, and V. Kumar, "Cooperative manipulation and transportation with aerial robots," Auton. Robot., vol. 30, pp. 73-86, 2011 .

[8] I. Maza, K. Kondak, M. Bernard, and A. Ollero, "Multi-UAV cooperation and control for load transportation and deployment," J. Intell. Robot. Syst., vol. 57, pp. 417-449, 2010.

[9] K. Sreenath, T. Lee, and V. Kumar, "Geometric control and differential flatness of a quadrotor UAV with a cable-suspended load," in Proc. $C D C, 2013$, pp. 2269-2274.

[10] T. Lee, K. Sreenath, and V. Kumar, "Geometric control of cooperating multiple quadrotor UAVs with a suspended load," in Proc. CDC, 2013, pp. 5510-5515.

[11] F. A. Goodarzi, D. Lee, and T. Lee, "Geometric stabilization of a quadrotor uav with a payload connected by flexible cable," in Proc. $A C C, 2014$, accepted.

[12] J. B. Hoagg and D. S. Bernstein, "Retrospective cost model reference adaptive control for nonminimum-phase systems," AIAA J. Guid. Contr. Dyn., vol. 35, pp. 1767-1786, 2012.

[13] J. B. Hoagg, M. A. Santillo, and D. S. Bernstein, "Discrete-time adaptive command following and disturbance rejection for minimum phase systems with unknown exogenous dynamics," IEEE Trans. Autom. Contr., vol. 53, pp. 912-928, 2008.

[14] M. A. Santillo and D. S. Bernstein, "Adaptive control based on retrospective cost optimization," AIAA J. Guid. Contr. Dyn., vol. 33, pp. 289-304, 2010.

[15] A. Morozov, J. B. Hoagg, and D. S. Bernstein, "Retrospective adaptive control of a planar multilink arm with nonminimum-phase zeros," in Proc. CDC, 2010, pp. 3706-3711.

[16] M. W. Isaacs, J. B. Hoagg, A. Morozov, and D. S. Bernstein, "A numerical study on controlling a nonlinear multilink arm using a retrospective cost model reference adaptive controller," in Proc. CDC 2011, pp. 8008-8013.

[17] G. Cruz and D. S. Bernstein, "Adaptive spacecraft attitude control with reaction wheel actuation," in Proc. ACC, 2013, pp. 4832-4837. 